\title{
Exploring developmental, functional, and evolutionary aspects of amphioxus sensory cells
}

\author{
Gouki Satoh \\ Graduate School of Life and Environmental Sciences, University of Tsukuba, Japan.
}

Corresponding address: Gouki Satoh, Graduate School of Life and Environmental Sciences, University of Tsukuba, 1-1-1 Tennodai, 305-8572 Tsukuba, Japan. E-mail: gouki@sakura.cc.tsukuba.ac.jp

Received: 2006.01.16; Accepted: 2006.04.14; Published: 2006.05.17

Amphioxus has neither elaborated brains nor definitive sensory organs, so that the two may have evolved in a mutually affecting manner and given rise to the forms seen in extant vertebrates. Clarifying the developmental and functional aspects of the amphioxus sensory system is thus pivotal for inferring the early evolution of vertebrates. Morphological studies have identified and classified amphioxus sensory cells; however, it is completely unknown whether the morphological classification makes sense in functional and evolutionary terms. Molecular markers, such as gene expression, are therefore indispensable for investigating the developmental and functional aspects of amphioxus sensory cells. This article reviews recent molecular studies on amphioxus sensory cells. Increasing evidence shows that the non-neural ectoderm of amphioxus can be subdivided into molecularly distinct subdomains by the combinatorial code of developmental cues involving the RA-dependent Hox code, suggesting that amphioxus epithelial sensory cells developed along positional information. This study focuses particularly on research involving the molecular phylogeny and expression of the seven-transmembrane, G protein-coupled receptor (GPCR) genes and discusses the usefulness of this information for characterizing the sensory cells of amphioxus.

Key words: vertebrate origins, sensory system, genome duplication, GPCR genes

\section{Introduction}

Amphioxus is sometimes referred to as an "acraniate", which is the counterpart of "craniate" and means headless animal. As discussed elsewhere, evidence of cephalization in protochordates can be seen both developmentally and anatomically. In particular, a comparison of developmental gene expression between amphioxus and vertebrates revealed evolutionarily conserved anteroposterior and dorsoventral patterns of the neural tubes $[1,2,3]$. These molecular correspondences at the embryonic and larval stages can sometimes be related to morphological outcomes seen in adult anatomy, as exemplified by the repeatedly promulgated homology between the vertebrate neurohypophysis and the infundibular organ of amphioxus $[4,5,6,7]$. Despite a series of detailed, EM-based neuroanatomical work by Lacalli and colleagues [8], there is no convincing evidence that amphioxus have no regions comparable to telencephalon-derived structures such as the cerebrum and olfactory bulb, suggesting that these are vertebrate innovations. This idea coincides with recent findings that the genes required for the formation of telencephalic structures in vertebrates show no comparable expression in amphioxus [9, 10]; further, the most anterior region of the amphioxus brain vesicle cannot be comparable to any region of vertebrates based on developmental gene expression patterns [11]. The vertebrate brain, according to the definition by Ayers [12], is an anterior portion of the neural tubes associated with organs of special senses. In other words, elaborations of the sensory organs and central nervous system undoubtedly underlie the appearance of considerably cephalized animal like vertebrates, and sensory organs and the central nervous system may have evolved in a mutually affecting manner, both functionally and embryologically. It is thus necessary to investigate the developmental and functional aspects of the amphioxus sensory system and to compare it with those of vertebrates.

Morphological descriptive works are beyond the scope of this review but have been reviewed by others $[13,14]$. Instead, this article focuses on recent molecular studies on the amphioxus epithelial sensory cells and photosensory cells. Gene expression analyses have gradually disclosed functional and developmental aspects of amphioxus sensory cells. Molecular phylogenetic analyses on the GPCR genes draw a picture of sensory system evolution in the chordate lineage, and, together with GPCR gene expression, make us possible to infer functional and evolutionary aspects of the amphioxus sensory cells. I introduce studies on the amphioxus opsin genes as a typical example of this line of research and discuss a possibility of G protein-coupled receptor (GPCR) genes for characterizing the amphioxus sensory cells. 


\section{Classification of epithelial sensory cells and their possible functions}

The epithelial sensory cells of amphioxus can be classified into several subtypes based on their morphologies [2, 15]. Amphioxus has numerous epithelial sensory cells (Fig. 1B), which possibly include mechanosensory and chemosensory cell populations. Possible sensory apparatuses are also existed in the velar tentacles (Fig. 1C) and oral cirri (Fig. 1D). However, to date, there is no evidence that the morphological classification of amphioxus sensory cells corresponds with their functional classification. Moreover, according to Holland and Holland [2], it is not easy to detect their evolutionary affinities with those derived from placodes, ectodermal thickenings that produce population of neurons in vertebrate development [1]. It appears to be difficult to investigate the functional and evolutionary aspects of the identified epithelial sensory cells by morphological observations alone; molecular markers, such as gene expressions, are necessary to characterize the epithelial sensory cells. Although the results of some gene expression studies have been reported recently, the number of such studies is sparse. To date, elav [16 ], Coe [17], trk [18], Hox1, Hox3, Hox4, Hox6 , ERR, and islet [19] genes are known to be expressed in the epithelial sensory cells. Satoh et al. [16] examined the expression pattern of the pan-neuronal marker gene elav from early neurulae to 48-h larvae. Elav expression possibly discloses most, if not all, of the nascent epithelial sensory cell population that becomes detectable in early neurulae. In the neurulae, the nascent epithelial sensory cell population was seen on the ventral side of the embryo. As development proceeded, signals became detectable in scattered cells located on the lateral sides of late neurulae. This expression pattern has also observed in [17] and [18]. It is therefore intriguing to ask whether ventrally located cells in early neurulae migrate to the lateral sides of the embryo. DiI labeling study has indicated that ventrally-located cells migrate towards lateral sides of the embryo [18]. In addition, based on the observation of Coe-positive cells during development, Mazet and colleagues [17] have discussed an evolutionary relationship between migrations of amphioxus epithelial sensory cells and vertebrate ectomesenchyme like neural crest-derived cells. However, explaining these observations do not always require a presumption that epithelial sensory cells of amphioxus can autonomously migrate during embryonic development. In my opinion, it is not hard to imagine that invagination of neuroectoderm that forms neural tube causes upward movement of the non-neural ectoderm, resulting in dorsal migration of ventrally-located epithelial sensory cell population. In order to address this issue, further experimental research will be required.

Figure 1. (A) Schematic representation of the rostral structures of amphioxus, a dorsal view. Adapted from [13]. Note that oral cirri (oc) have been simplified. not; notochord, nt; neural tube, vt; velar tentacle, wo; wheel organ. (B) - (D) Scanning electron micrographs showing body surface with numerous corolla-like structures of epithelial sensory cells (B), velar tentacle with possible sensory apparatus called sensory papilla (arrow) (C), and possible sensory apparatuses located on the lateral sides of each oral cirrus (arrows) (D). Scale bars respectively represent $25 \mu \mathrm{m}$ (B) and $10 \mu \mathrm{m}$ (C).
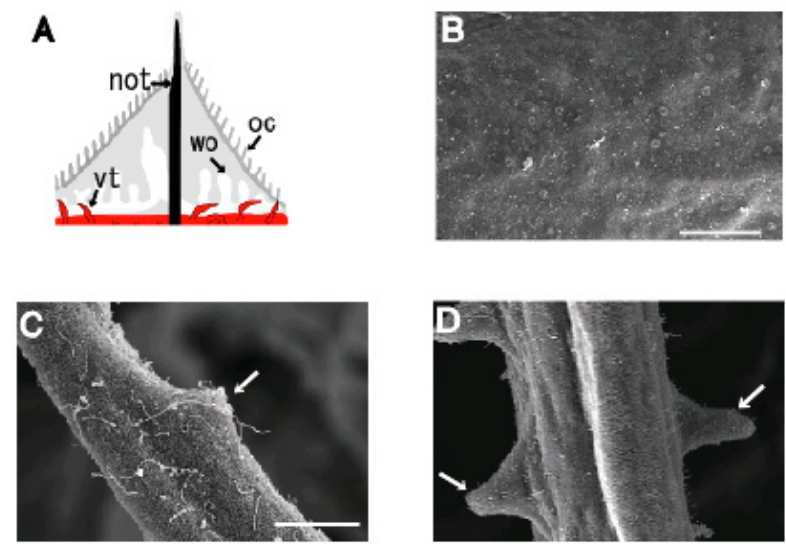

Recent reports have highlighted the novel mechanism governing the development of amphioxus epithelial sensory cells. Schubert et al. [19] reported that retinoic acid (RA)-dependent anteroposterior patterning is relevant for the subdivision of epithelial sensory cells. In fact, collinear and segmental expression of Hox genes has been observed not only in the neural tube [20] but also in the general ectoderm [19]. Benito-Gutierrez et al. [18] isolated an amphioxus cognate of the trk receptor gene and examined its expression. According to their observations, AmphiTrk is expressed in both primary and secondary epithelial sensory cells, and this expression precedes that of elav. As elav is involved in neuronal differentiation such as axonal outgrowth [21, 22], the trk signaling system is likely involved in the early development of amphioxus epithelial sensory cells. Interestingly, it seems that there are fewer AmphiTrk-positive cells than elav-positive cells. Likewise, the number of cells that express AmphiCoe, a COE/EBF family gene with vertebrate counterparts involved in the placode-derived sensory cells, seems to be fewer than those expressing elav [17]. These findings suggest that the combinatorial code of developmental cues involving the Hox code may subdivide amphioxus epithelial sensory cells as a whole into molecularly distinct subdomains. Although the expression of these genes does not directly shed light on the functional aspects of the identified epithelial sensory cells, it is noteworthy that mice with mutated trk genes show defects in their mechanosensory cells [23]. Furthermore, immunohistochemical research has indicated the existence of trk-like substances in the fish lateral line $[24,25]$.

To gain insight into the functional modality of epithelial sensory cells of amphioxus, Satoh [26] tried to characterize these cells based on the molecular 
phylogeny and expression of the $G$ protein-coupled receptor (GPCR) genes. The GPCR family in vertebrates contains multiple copies of gustatory and olfactory receptor genes and photosensitive opsin genes. Thus, identifying the amphioxus counterparts of these GPCR genes may be useful in disclosing the functions of the identified sensory neurons. It may even be possible to infer the causal relationship between the elaboration of sensory modalities and large-scale genomic duplication thought to have taken place in a lineage leading to the vertebrates [27, 28, 29, 30], although Horton and colleagues proposed that molecular phylogenetic analyses are insufficient for dating genomic duplication events in the vertebrate phylogeny [31]. In particular, multiple copies of vertebrate olfactory receptor (OR) genes are arrayed throughout the genome, yielding 100 genes in teleosts and 1000 genes in rodents [32]. Each vertebrate olfactory sensory neuron selectively chooses only one allele of the receptor gene from a vast array of genes, and vertebrate ORs predominantly lack introns. Gentles and Karlin [33] proposed that the mRNA of an ancestral olfactory receptor gene was reverse-transcribed and incorporated in the genome, resulting in the early template of the intronless ORs.

An intronless GPCR gene found in the amphioxus genome showed weak sequence similarity to the vertebrate ORs. Although molecular phylogenetic analysis assigned this gene to a sister group of the vertebrate ORs [26], conclusive phylogenetic assignments should await genome-wide characterization of the amphioxus GPCR genes. In addition, there is no evidence of the existence of other GPCR genes located in close proximity to the intronless GPCR gene [26]. The ORs of the zebrafish are evenly interspaced by about $1-\mathrm{kb}$ non-coding regions [34], suggesting that duplication of the olfactory-related GPCR genes may have occurred in a lineage leading to the vertebrates or gnathostomes. cDNAs of lamprey ORs have been characterized [35, 36], whereas the number of ORs in the lamprey genome remains unknown; more importantly, the genomic organization of the lamprey ORs (i.e., whether they have introns or not) remains to be investigated. For these reasons, it is so far impossible to date precisely the massive expansion of the ORs in the vertebrate phylogeny.

The expression of the amphioxus GPCR gene was seen in the bipolar neurons embedded within the rostral epithelium (Fig. 2). If this expression domain correspond with a region thought to be comparable to the vertebrate nasal epithelium based on the larval expression of the Pax6 [37], BMP2/4 [38], and msx [39] genes, the GPCR-positive bipolar neurons can be seen as chemosensory cell populations. Taking neuroanatomical data into consideration, neurites of the GPCR-positive epithelial sensory cells seem to constitute a nerve fiber called the anterodorsal nerve. The anterodorsal nerve is composed of two major branches, the dorsal branch and ventral branch [15]. It is therefore possible that the dorsal and ventral branches respectively correspond to the dorsally located and ventrally located GPCR-positive cell populations (Fig. 2) (see also [26] and [40]). Furthermore, there is another nerve located rostrally in relation to the anterodorsal nerve, the so-called rostral nerve. The rostral nerve terminates at the rostral tip, a GPCR-negative region (Fig. 2). Collectively, the rostral epithelium of amphioxus can be functionally subdivided into subdomains, and each peripheral nerve is likely to correspond to the regionalization of the epithelial sensory cells. However, it remains to address whether the regionalization seen in the rostral epithelium of amphioxus makes any sense in functional or evolutionary context. Nevertheless, it is worth to note that the rostral epithelium of appendicularian, the earliest branch of the urochordates [41], can be subdivided into molecularly distinct subdomains comparable to the vertebrate hypophyseal, olfactory, and otic placodes [42], despite no molecular evidences of such regionalization in other tunicates like ascidians except for putative hypophyseal placode counterpart (for review, see [43]).

Figure 2. Schematic representation showing expression of Amphi-GPCR1 (red) in relation to peripheral nerves, the so-called anterodorsal nerve (adn) and rostral nerve (rn). The anterodorsal nerve is composed of two major branches, a dorsal branch $(\mathrm{db})$ and ventral branch $(\mathrm{vb})$ [15]. bv; brain vesicle, not; notochord.

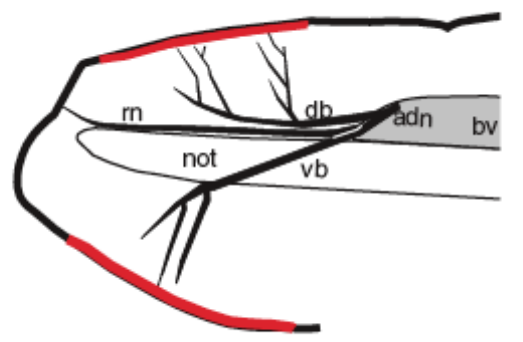

\section{Molecular phylogeny of rhabdomeric and ciliary opsins shed lights on the evolutionary history of rhabdomeric and ciliary photosensory cells}

The photosensory cells of metazoans can be subdivided into two types, rhabdomeric cells and ciliary cells, based on their morphologies. Molecular phylogenetic analysis has revealed that opsin genes can also be subdivided into two major types, rhabdomeric and ciliary opsins [44] (Fig. 3). Opsin proteins possess an evolutionarily conserved lysine residue, located on the seventh helix, that binds to the 11-cis-retinal to constitute visual pigments. Rhabdomeric cells contain rhabdomeric opsins in their microvillar projections, whereas ciliary cells contain ciliary opsins in the discs formed by invagination of the cilium membrane. Rhabdomeric cells are predominantly seen in invertebrate eyes, and there are no photosensory cells that can morphologically be 
regarded as rhabdomeric cells in vertebrates. Nevertheless, it has found that the melanopsin gene, a vertebrate cognate of the rhabdomeric opsin gene, is expressed in photosensitive retinal ganglion cells (RGCs) [45]. Furthermore, the cytodifferentiation of RGCs is regulated by the $R x$ gene, similar to the regulation of invertebrate rhabdomeric cells [46, 47]. These findings have led to speculation that the "missing" rhabdomeric cells of vertebrates may have survived as RGCs.

Although ciliary cells are well known as cones and rods in the vertebrate retina and photosensory cells of the pineal, some invertebrates such as ragworms [46] and amphioxus $[48,49,50,51]$ also have both ciliary and rhabdomeric cells. It had been unknown whether invertebrates possess ciliary opsin genes closely related to those of the vertebrates, and, in fact, no ciliary opsin genes exist in the C. elegans and Drosophila genomes. However, Arendt et al. [44] recently isolated a cognate of the ciliary opsin gene from ragworm, with expression seen in the ciliary cells. In addition, Velarde et al. [52] and Spaethe and Briscoe [53] identified the ciliary opsin gene in honeybee and mosquito, respectively. These findings seem to support the idea that ancestral bilateria possessed both ciliary and rhabdomeric opsins and, accordingly, ciliary and rhabdomeric cells. In contrast, Salvini-Plawen and Mayr [54] postulated that the photosensory cells of metazoans arose independently in phylogenies.

Figure 3. Molecular phylogenetic tree based on the maximum-likelihood method, showing the phylogenetic relationships of ciliary and Go-coupled opsin proteins. The tree was rooted with rh4 opsin of Drosophila. Note that branch length is not proportional to the genetic distance. The numbers at each node are the percentage confidence values of the ML tree

To infer the evolutionary relationship between the photosensory cells of invertebrates and vertebrates, molecular phylogenetic analysis regarding amphioxus opsin genes is indispensable. It is particularly important to examine whether a ciliary and
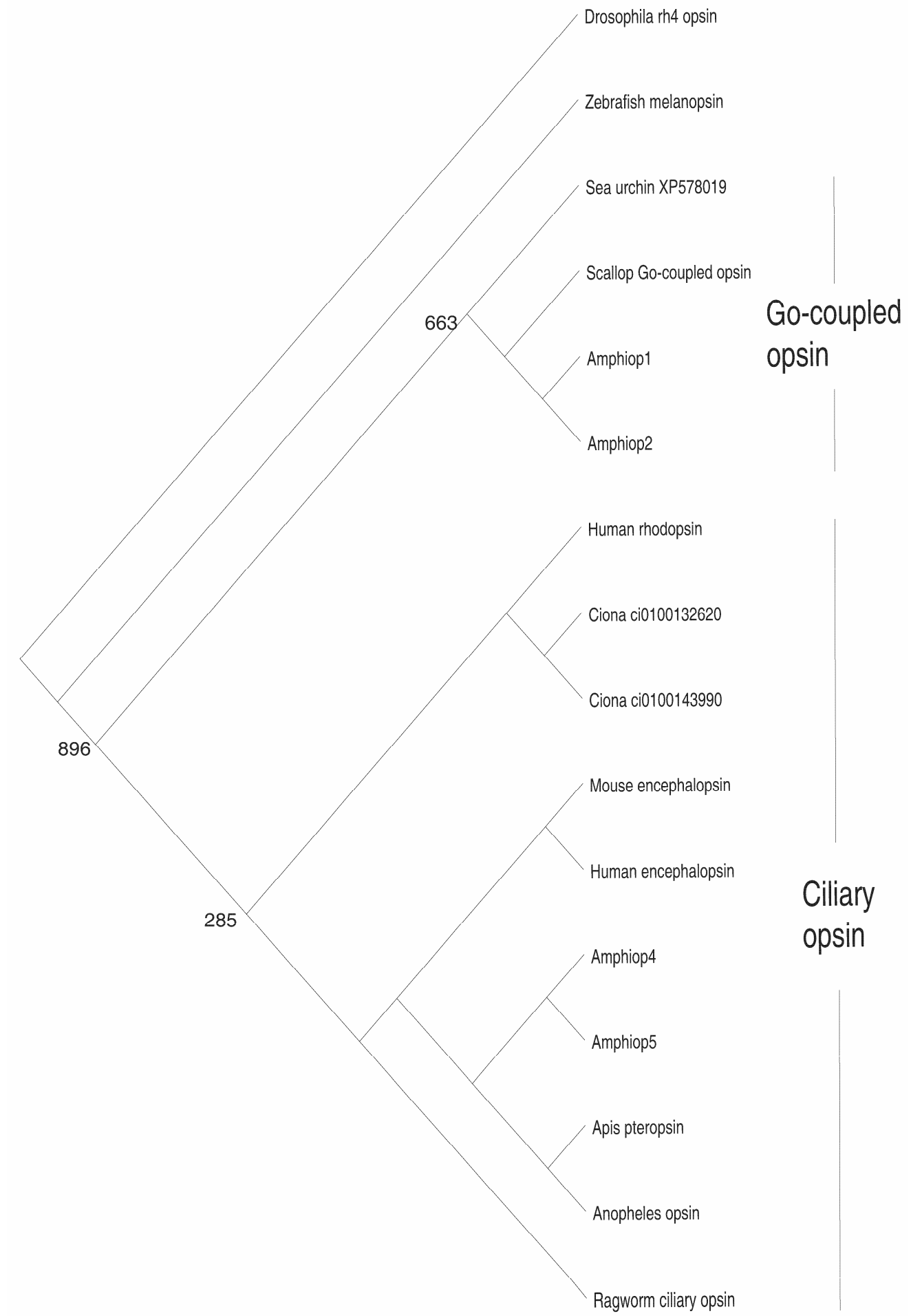

rhabodomeric dichotomy has been maintained even in 
the chordate lineage. Koyanagi et al. [55] reported that the amphioxus counterpart of melanopsin, a rhabdomeric opsin, possesses a photochemical property that is quite similar to that of the invertebrate opsin gene and that its expression is seen in Hesse's eye cups and Joseph cells, which clearly have rhabdomeric morphologies [48, 51]. These results, together with the monophyly of the rhabdomeric opsin gene and the Gaq gene [44], support a homology between vertebrate RGCs and invertebrate rhabdomeric cells, although this must be corroborated by developmental gene expression studies in the future.

In contrast to that of the rhabdomeric cells, the evolutionary histories of ciliary cells and ciliary opsin genes appear to be more complex. Molecular phylogenetic analysis has assigned Amphiop5 as a ciliary opsin, whereas Amphiop1 clustered with so-called Go-coupled opsin (Fig. 3). Amphiop1 is expressed in the photosensory cells of larval frontal eye, while Amphiop5 is expressed in the frontal eye of adults (Satoh et al., unpublished observation). Amphiop1 protein has been shown to bind to 11-cis-retinal [56], suggesting that Amphiop1 is involved in photodetection in the frontal eye of amphioxus larvae, which show diurnal vertical migration in the water column [57]. The Go-coupled opsin gene of the scallop is also expressed in ciliary cells [58], so that Go-coupled opsin can be seen as a part of the ciliary opsin. Consequently, ciliary opsin in broad sense is likely composed from two distinct evolutionary lineages. More importantly, the Go-coupled opsin group is devoid of vertebrate counterparts, implying that Go-coupled opsin became extinct in the vertebrate lineage. If so, highly diversified ciliary opsin genes in vertebrates have derived from very limited number of early templates via repeated gene duplication. In line with this notion, Nordstrom and colleagues [59] postulated that the majority of the genes involved in the phototransduction pathways of vertebrates may have arisen by an extensive gene duplication event in early vertebrate evolution. Taken together, an increase in the repertoire of phototransduction genes, including opsin, underlie the evolution of the functionally diverse ciliary cells seen in the vertebrate retina.

\section{Molecular evolution of GPCR genes and characterization of the sensory cells: A problem}

As discussed above, the expression of GPCR genes appears to be a useful marker for disclosing the functional and evolutionary aspects of amphioxus sensory cells. Studies on opsin gene expression are a typical example of this line of research. A clear distinction between ciliary and rhabdomeric opsin genes across the bilaterian phylogeny suggests the possibility that the molecular phylogenetic classification of GPCR genes may agree with the functional and morphological classifications of amphioxus sensory cells in which GPCR genes are expressed. However, the molecular phylogeny of
GPCR genes is complex and requires careful analysis because of the high substitution rates and the independent gain and loss of GPCR genes. For example, it is difficult to identify a clear evolutionary affinity between the chemosensory receptor genes of $C$. elegans and Drosophila based on sequence comparisons [60], suggesting that these genes have formed independently. This raises concerns regarding surveys of the chemosensory GPCR genes in the amphioxus genome. It is possible that amphioxus has chemosensory receptor genes whose sequences are quite different from those of vertebrate ORs or gustatory receptor genes. Thus, in the post-genomic era, a comprehensive analysis of amphioxus GPCR genes will be necessary. In addition to the classification of the amphioxus GPCR genes as a whole based on molecular phylogenetic analysis, investigations of the genomic organization and expression analyses will also be required to determine the functional modalities of sensory cells and to date massive expansion of the GPCR genes in early vertebrate evolution.

\section{Acknowledgments}

Gouki Satoh is a postdoctoral research fellow of the Japan Society for the Promotion of Sciences (JSPS) and is supported by a research grant (17-7109) from the Ministry of Education, Culture, Sports, Science, and Technology (MEXT) of Japan.

\section{Conflict of interest}

The author has declared that no conflict of interest exists.

\section{References}

1. Holland LZ, Holland ND. Chordate origins of the vertebrate central nervous system. Curr Opin Neurobiol 1999; 9: 596-602.

2. Holland LZ, Holland ND. Evolution of neural crest and placodes: amphioxus as a model for the ancestral vertebrates? J Anat 2001; 199: 85-98.

3. Shimeld SM, Holland ND. Amphioxus molecular biology: insight into vertebrate evolution and developmental mechanisms. Can J Zool 2005; 83: 90-100.

4. Olsson R, Yulis R, Rodriguez EM. The infundibular organ of the lancelet (Branchiostoma lanceolatum, Acrania): An immunohistochemical study. Cell Tissue Res 1994; 277: 107-113.

5. Gorbman A. Olfactory origins and the evolution of the brain-pituitary endocrine system: Facts and speculation. Gen Comp Endocrinol 1995; 97: 171-178.

6. Yasui K, Zhang S, Uemura M, Saiga H. Left-right asymmetric expression of BbPtx, a Ptx-related gene, in a lancelet species and the developmental left-sidedness in deuterostomes. Development 2000; 127: 187-195.

7. Boorman CJ, Shimeld SM. Pitx homeobox gene in Ciona and amphioxus show left-right asymmetry is a conserved chordate character and defines the ascidian adenohypophysis. Evol Dev 2002; 4: 354-365.

8. Northcutt RG. The new head hypothesis revisited. J Exp Zool (Mol Dev Evol) 2005; 304B: 274-297.

9. Horton AC, Gibson-Brown JJ. Evolution of developmental functions by Eomesodermin, T-Brain1, Tbx21 subfamily of T-box genes: insights from amphioxus. J Exp Zool (Mol Dev Evol) 2002; 294: 112-121. 
10. Satoh G, Takeuchi JK, Yasui K, Tagawa K, Saiga H, Zhang P, Satoh N. Amphi-Eomes/Tbr1: An amphioxus cognate of vertebrate Eomesodermin and T-Brain1 genes whose expression reveals evolutionarily distinct domain in amphioxus development. J Exp Zool 2002; 294:136-145.

11. Mazet F, Shimeld SM. The evolution of chordate neural segmentation. Dev Biol 2002; 251: 258-270.

12. Ayers H. Concerning vertebrate cephalogenesis. J Morphol 1890; 4: 221-245.

13. Frantz V. Haut, Sinnesorogane und Nervensystem der Akranier. (Fauna et Anatomia ceylancia, Nr.13). Jenais Zeit Naturw 1923; 59: 401-526.

14. Ruppert EE. Cephalochordata (Acrania). In: Harrison FW, Ruppert EE, editors. Microscopic anatomy of invertebrates vol 15. New York: Wiley Liss, 1997: 349-504

15. Lacalli TC. Sensory systems in amphioxus: a window on the ancestral chordate condition. Brain Behav Evol 2004; 64: 148-162.

16. Satoh G, Wang Y, Zhang P, Satoh N. Early development of amphioxus nervous system with special reference to segmental cell organization and putative sensory cell precursors: A study based on the expression of pan-neuronal marker gene Hu/elav. J Exp Zool (Mol Dev Evol) 2001; 291: 354-364.

17. Mazet F, Masood S, Luke GN, Holland ND, Shimeld SM. Expression of AmphiCoe, an amphioxus COE/EBF gene, in the developing central nervous system and epidermal sensory neurons. genesis 2004; 38: 58-65.

18. Benito-Gutierrez E, Nake C, Llovera M, Comella JX, Garcia-Fernandez J. The single AmphiTrk receptor highlights increased complexity of neurotrophin signaling invertebrates and suggests an early role in developing sensory neuroepidermal cells. Development 2005; 132: 2191-2202.

19. Schubert M, Yu J-K, Holland ND, Escriva H, Laudet V, Holland LZ. Retinoic acid signaling acts via Hox1 to establish the posterior limit of the pharynx in the chordate amphioxus. Development 2005; 132: 61-73.

20. Wada H, Garcia-Fernandez J, Holland PWH. Colinear and segmental expression of amphioxus Hox genes. Dev Biol 1999; 213: 131-141.

21. Aranda-Abreu GE, Gehar L, Chung S, Furneaux H, Ginzburg I. Embryonic-lethal abnormall vision-like RNA binding proteins regulate neurite outgrowth and tau expression in PC12 cells. J Neurosci 1997; 19: 6907-6917.

22. Perrone-Bizzozelo N, Bolognani F. Role of $\mathrm{HuD}$ and other RNA-binding proteins in neural development and plasticity. J Neurosci Res 2002; 68: 121-126.

23. Stucky CL, DeChiara T, Lindsay RM, Yancopoulos GD, Koltzenburg M. Neurotrophin4 is required for the survival of a subclasss of hair follicle receptors. J Neurosci 1998; 18: 7040-7046.

24. Hannestad J, Marino F, Garmana A, Catania S, Abbate F, Ciriaco E, Vega JA. Trk neurotrophin receptor-like proteins in the teleost Dicentrachun labrax. Cell Tissue Res 2000; 300: 1-9.

25. Germana A, Catania S, Cavallaro M, Gonzalez-Martinez T, Ciriaco F, Hannestad J, Vega JA. Immunohistochemical localization of BDNF-, TrkB- and TrkA-like proteins in the teleost lateral line system. J Anat 2002; 200: 477-485.

26. Satoh G. Characterization of novel GPCR gene coding locus in amphioxus genome: gene structure, expression and phylogenetic analysis with implications for its involvement in chemoreception. genesis 2005; 41: 47-57.

27. Ohno S. Evolution by gene duplication. New York: Springer-Verlag, 1971.

28. Holland PWH. Major transitions in animal evolution: A developmental genetic perspective. Am Zool 1998; 38 : 829-842.
29. Panopoulou G, Hennig S, Groth D, Krause A, Poustka AJ, Herwig R, Vingron $M$, Lehrach $H$. New evidence for genome-wide duplications at the origin of vertebrates using gene set and completed animal genomes. Genome Res 2002; 13 1056-1066.

30. Robinson-Rechavi M, Boussau B, Laudet V. Phylogenetic dating and characterization of gene duplications in vertebrates: the cartilaginous fish reference. Mol Biol Evol 2004; 21: 580-586.

31. Horton AC, Mahadevan NR, Ruvinsky I, Gibson-Brown JJ. Phylogenetic analyses alone are insufficient to determine whether genome duplication(s) occurred during vertebrate evolution. J Exp Zool (Mol Dev Evol) 2003; 299: 41-53.

32. Mombaerts P. Seven-transmembrane proteins as odorant and chemosensory receptors. Science 1999; 286: 707-711.

33. Gentles AJ, Karlin S. Why human G protein-coupled receptors predominantly intronless? Trends Genet 1999; 15: 47-49.

34. Kratz E, Dugas JC, Ngai J. Odorant receptor gene regulation: implication from genomic organization. Trends Genet 2002; 18: 29-34.

35. Berghard A, Dryer L. A novel family of ancient vertebrate odorant receptors. J Neurobiol 1998; 37: 383-392.

36. Freitag J, Beck A, Ludwig G, von Buchholts L, Breer H. On the origin of the olfactory receptor family: receptors genes of the jawless fish (Lampetra fluviatilis). Gene 1999; 226: 165-174.

37. Glardon S, Holland LZ, Gehring WJ, Holland ND. Isolation and developmental expression of the amphioxus Pax-6 (AmphiPax-6): insights into eye and photoreceptor evolution. Development 1998; 125: 2701-2710.

38. Panopoulou GD, Clark MD, Hollanbd LZ, Lehrach H, Holland ND. AmphiBMP2/4, an amphioxus bone morphogenetic protein closely related to Drosophila decapentaplegic and vertebrate BMP2 and BMP4: insights into evolution of dorsoventral axis specification. Dev Dyn 1998; 213: 130-139.

39. Sharman AC, Shimeld SM, Holland PWH. An amphioxus Msx gene expressed predominantly in the dorsal neural tube. Dev Genes Evol 1999; 209: 260-263.

40. Satoh G. A trajectory of increasing activeity and the elaborateion of chemosensory modality: a new perspective on the vertebrate origins. Zool Sci 2005; 22: 613-626.

41. Wada H. Evolutionary history of free-swimming and sessile lifestyles in urochordates as deduced from 18S rDNA molecular phylogeny. Mol Biol Evol 1998; 15: 1189-1194.

42. Bassham S, Postlethwait JH. The evolutionary history of placodes: A molecular genetic investigation of the larvacean urochordate Oikopleura dioica. Development 2005; 132: 4259-4272.

43. Schlosser G. Evolutionary origins of vertebrate placodes: insight from developmental studies and from comparisons with other deuterostomes. J Exp Zool (Mol Dev Evol) 2005; 304: 347-399.

44. Arendt D. Evolution of eyes and photoreceptor cell types. Int J Dev Biol 2003; 47: 563-571.

45. Jenkins A, Munoz M, Tarttelin EE, Bellingham J, Foster RG, Hankins MW. VA opsin, melanopsin, and an inherent light response within retinal interneurons. Curr Biol 2003; 13: 1269-1278.

46. Arendt D, Tessmar-Raible K, Snyman H, Dorresteijn AW, Wittbrodt J. Ciliary photoreceptors with a vertebrate-type opsin in an invertebrate brain. Science 2004; 306: 869-871.

47. BaileyTJ, El-Hodiri H, Zhang L, Shah R, Mathers PH, Jamrich $\mathrm{M}$. Regulation of vertebrate eye development by Rx genes. Int J Dev Biol 2004; 48: 761-770.

48. Watanabe T, Yoshida M. Morphological and histochemical studies on Joseph cells of amphioxus, Branchiostoma belcheri, Gray. Exp Biol. 1986;46: 67-73. 
49. Ruiz S, Anadon I. The fine structure of lamellate cells in the brain of amphioxus (Branchiostoma lanceolatum, Cephalochordata). Cell Tiss Res 1991; 263: 597-600.

50. Lacalli TC, Holland ND, West JE. Landmarks in the anterior central nervous system of amphioxus larvae. Phil Trans R Soc Lond B 1994; 344: 165-185.

51. Castro A, Becerra M, Manso MJ, Sherwood NM, Anadon R. Anatomy of the Hesse photoreceptor cell axonal system in the central nervous system of amphioxus. J Comp Neurol 2006; 494: 54-62.

52. Velarde RA, Sauer CD, Walden KK, Fahrbach SE, Robertson HM. Pteropsin: a vertebrate-like non-visual opsin expressed in the honeybee brain. Insect Biochem Mol Biol 2005; 35: 1367-1377.

53. Spaethe J, Briscoe AD. Early duplication and functional diversification of the opsin gene family in insects. Mol Biol Evol 2004; 21: 1583-1594.

54. Salvini-Plawen LV, Mayr E. On the evolution of photoreceptors and eyes. Evol Biol 1977; 10: 207-263.

55. Koyanagi M, Kubokawa K, Tsukamoto H, Shichida Y, Terakita A. Chaphalochordate melanopsin: evolutionary linkage between invertebrate visual cells and vertebrate photoreceptive retinal ganglion cells. Curr Biol 2005; 15: 1065-1069.

56. Koyanagi M, Kubokawa K, Tsukamoto H, Shichida Y. Amphioxus homologs of Go-coupled rhodopsin and peropsin having 11-cis- and all-trans- retinals as their chromophores. FEBS Lett 2002; 531: 525-528.

57. Wickstead JH, Bone Q. Ecology of acraniate larvae. Nature 1959; 184: 1849-1851.

58. Kojima D, Terakita A, Ishikawa T, Tsukahara T, Maeda A, Shichida Y. A novel Go-mediated phototransduction cascade in scallop visual cells. J Biol Chem 1997; 272: 22979-22982.

59. Nordstrom K, Larsson TA, Larhammer D. Extensive duplications of phototransduction genes in early vertebrate evolution correlate with block (chromosome) duplications. Genomics 2004; 83: 852-872.

60. Krieger J, Breer H. Ofactory reception in invertebrates. Science 1999; 286: 720-723.

\section{Author biography}

Gouki Satoh obtained Ph. D. degree from Kyoto University in 2003. As a predoctoral research fellow of the Japan Society for the Promotion of Science (JSPS) (2001-2003), he focused on the embryonic development and gene expression patterns of ascidians and amphioxus for comparisons with vertebrates. Currently, he is a postdoctoral research fellow of the JSPS and investigates molecular evolution of GPCR genes to disclose an evolutionary aspect of the amphioxus sensory system. 